\title{
Anemia in Pregnancy
}

Chowdhury $\mathrm{S}^{1}$, Rahman $\mathrm{M}^{2}$, Moniruddin $\mathrm{ABM}^{3}$

\begin{abstract}
Anemia is the commonest hematological disorder that occurs in pregnancy. According to the recent standard laid down by 'WHO', anemia is present when the Hemoglobin (Hb) concentration in the peripheral blood is $11 \mathrm{gm} / \mathrm{dl}$ or less. The most common cause of anemia in pregnancy is lack of iron. Less often, it is caused by folic acid deficiency. In some populations, $80 \%$ of pregnant women are anemic. Those most at risk are women from low socio-economic groups and teenagers. Anemia is diagnosed by estimating the hemoglobin concentration and examining a peripheral blood smear for the characteristic red blood cell changes. Iron and folate supplementation is indicated during pregnancy to prevent the complications.
\end{abstract}

Key words : Erythropoiesis, Puerperium, APH, PPH, ANC, $L B W, I U D$.

\section{Introduction}

Anemia is a lack of functioning red blood cells (RBCs) that leads to a lack of oxygen-carrying ability, causing unusual complications during life time ${ }^{1}$. These RBCs are produced in the bone marrow. They have a life expectancy of about 120 days. Among other things, the body needs iron, vitamin $\mathrm{B}_{12}$ \& folic acid for erythropoiesis. If there is a lack of one or more of these ingredients or there is an increased loss of RBCs, anemia develops. Any patient with a $\mathrm{Hb}$ of less than 11 $\mathrm{gm} / \mathrm{dl}$ to $11.5 \mathrm{gm} / \mathrm{dl}$ at the start of pregnancy will be treated as anemic. The reason is that as the pregnancy progresses, the blood is diluted and the woman will eventually become anemic. The dilution of blood in pregnancy is a natural process and starts at approximately at the eighth week of pregnancy and progresses until the 32nd to 34th week of pregnancy $^{2}$.

\section{Incidence}

In tropical countries, the incidence of anemia ${ }^{3}$ in pregnancy is about $40-80 \%$.

In developed countries, it ranges between $10-20 \%$.

It is responsibe for $20 \%$ of maternal death in developing countries.

1. Corresponding Author: Salma Chowdhury MBBS, DGO Senior Consultant (Gynae)

Central Police Hospital, Rajarbag, Dhaka

Email: dr.salmachowdhury@yahoo.com

2. Mizanur Rahman MBBS, FCPS

Associate Professor of Surgery

Faridpur Medical College, Faridpur

3. ABM Moniruddin MBBS, FCPS

Professor of Surgery

City Medical College, Itahata, Gazipur

\section{Classification of anemia in pregnancy}

Grossly classified into two types ${ }^{4}$ :

(A) Pathological anemia in pregnancy.

(B) Physiological anemia in pregnancy.

(A) Pathological Anemia is further sub-classified into

1. Deficiency Anemia, e. g.,

-Iron deficiency

-Folic acid deficiency

-B12 deficiency

-Protein deficiency

\section{Hemorrigic:}

Acute hemorrhagic: Following bleeding in early month of pregnancy or APH

Chronic hemorrhagic: as by hookworm infestation, GI (gastrointestinal) bleeding.

1. Hereditary:

Thalassemias - Haemolobinopathies.

Hereditary hemolytic anemia-RBCs defects.

2. Bone Marrow insufficiency: as by radiation, marrow suppressant drugs.

3. Anemia of infection - as by malaria tuberculosis

4. Chronic diseases : as in nephropathies \& neoplastic disorders.

It is noteworthy that obstetricians are concerned with two common types of anemia. They are :

5. Deficiency anemia,

6. Haemorrhagic anemia

It has been found there is increased prevalence of anemia in pregnancy in tropical countries.

This is due to

a. Faulty dietary habit,

b. Faulty absorption mechanism,

c. More iron loss due to sweating and repeated pregnancy at short interval; prolonged period of lactation,

d. Infection : Chronic malaria, tuberculosis,

e. Excess demand of iron : pregnancy is an iron deficit state.

\section{(B) Physiological Anemia}

During pregnancy there is disproportionate increase in plasma volume upto $50 \%$, RBC $33 \%$ and $\mathrm{Hb} 18-20 \%$ mass. In addition there is marked demand of extra iron during 
pregnancy especially in the second half of pregnancy. So, physiological anemia is due to combined effect of hemodialution $\&$ negative iron balance.

Criteria of Physiological Anemia include ${ }^{5}$

- $\mathrm{Hb} \%$ - 10 gm $\mathrm{r}$ less,

- R.B.C - 3.5 million $/ \mathrm{mm}^{3}$,

- P.C.V - 30\%,

- PBF - Normal morphology with central pallor.

Clinical features of iron deficiency Anemia depends more on the degree of anemia. Symptoms of anemia include lassitude, feeling of exhaustion, weakness, anorexia, indigestion, palpitation, swelling legs Signs of anemia include pallor, glossitis, Stomatitis, edema legs, soft systolic murmur in mitral area. Investigations are done to detect the degree of anemia, the type of anemia the cause of anemia.

To ascertain the degree of anemia one must look for $\mathrm{Hb} \%$, RBC count, PCV (Packed Cell Volume). Mild anemia means Hb- 8-10 gm\%; Moderate- less than 7-8 gm\%; Severe - Less than $7 \mathrm{gm} \%$.

To determine type of anemia one must examine the PBF (Peripheral Blood Film), hematological indcies like MCV, $\mathrm{MCH}, \mathrm{MCHC}$, etc.

A typical iron deficiency anemia shows the flowing blood values:

- Hb-less than $10 \mathrm{gm} \%$

- $\mathrm{RBC}$ - less 4 million/ mm3

- PCV - less than $30 \%$

- $\mathrm{MCHC}$ - Less than $30 \%$

- MCV - less than 75\% micro mole m3 (meter cube)

- $\mathrm{MCH}-$ less than $25 \mathrm{pg}$.

Serum iron is usally below 30 micro gram/ $100 \mathrm{ml}$. Total iron binding capacity increases to $400 \mathrm{micro}$ gram $/ 100 \mathrm{ml}$. Serum ferritin falls below 15 micro gm/L.

To find out the cause of anemia, the physician should carefully follow the basic protocols.

- History taking,

- Physical examination,

- Routine examination of stool to detect helminthes or occult blood,

- Urine is examined for the protein, sugar and pus cells,

- X ray chest in suspected cases of pulmonary tuberculosis; but in case not responding to therapy, bone marrow study should be undertaken.

- Blood for PBF \& malarial parasites,

- Kidney function tests like BUN \& s. creatinine, etc.

\section{Differential Diagnosis of deficiency anemias in pregnancy: 6}

- Infection,

- Nephritis,

- Hemoglobinnopathies.

\section{Treatment of anemia in Pregnancy: ${ }^{7}$}

- Prophylactic

- Curative Prophylaxis includes

- Avoidance of frequent child birth by proper family planning method.

Dietary prescription: Realistic balanced diet rich in iron and portion like liver, meat, eggs, green vegetable etc. Adequate treatment should be instituted to eradicate hook worm infestation, control of dysentery, malaria, nephropathies \& excision of bleeding piles. Hb level should be estimated at the $1^{\text {st }} \mathrm{ANV}$ and $30^{\text {th }}$ and finally at $36^{\text {th }}$ week.

Curative treatment:

- Hospitalisation, if $\mathrm{Hb}$ level is below 7.5 gram percent.

General treatment:

- Diet - balanced diet rich in protein, vitamins and iron

- Antibiotic for infective focuses, if any.

Specific Therapy as needed:

- Oral,

- Parental,

- Blood transfusion.

Depending on

- Severity of anemia,

- Duration of pregnancy,

- Associate complicating factor.

Iron Therapy:

Parenteral -

1. Intravenous,

a. Total dose infusion,

b. Multidose infusion,

2. Intramuscular:

- Iron dexran,

- Iron sorbital.

Estimation of total requirement:

$0.3 \mathrm{X} \mathrm{W}$ ( $100-\mathrm{Hb} \%$ ) gm of elemental iron + addition of $50 \%$. Improvement is expected withon 3-4 weeks.

Advantage of blood transfusion: ${ }^{8}$

- Increased O2 carrying capacity,

- Hb from heamolysed RBC,

- Stimulating by erythropoietin, 
- Supplies natural content of blood antibodies,

- Improvement is expected after 3 days.

Precaution:

- To prevent circulatory over load,

- Antihistamine for allergic episodes,

- Diuretic injection 20 mg Frusemide,

- Careful observation of respiration \& crepitations at the lung bases.

Place of blood transformation :

Indications:

- To correct blood loss,

- To combat PPH,

- Beyond 36 weeks and severe anemia, refectory anemia.

Quality and quantity of blood:

- Fresh and properly typed, grouped and cross matched; only PCV are transfused.

- Quantity 80-100 $\mathrm{ml}$ at a time interval after 24 hours for circulating readjustment.

\section{Complications of severe anemia: ${ }^{9}$}

During pregnancy-

- Pre-eclampisa,

- Recurrent infection,

- Heart failure,

- Preterm labour,

During labor-

- Uterine inertia,

- PPH,

- Cardiac failure,

- Shock.

During puerperium:

- Puerperal sepsis,

- Sub-involution,

- Failing lactation.

Management during labor:

In $1^{\text {st }}$ stage-

- Patient. should be in bed and should be in a position comfortable to her,

- Arrangement for oxygen,

- Aseptic condition is to me maintained.

In $2^{\text {nd }}$ stage-

- Prophylactic low force/ vacuum delivery.
In $3^{\text {rd }}$ stage-

- Active management.

Prophylactic antibiotic to prevent infection

- Iron therapy for at least 3 months following delivery.

Risk periods:

- At 30-32 weeks of pregnancy,

- During labor,

- Immediately following delivery.

\section{Prognosis: ${ }^{10}$}

Maternal aspect-

1. If detected early \& proper treatment is instituted, anemia improves promptly;

2. Substantial chances of recurrence in next pregnancy,

3. Contributes to about $2-\%$ maternal death in developing countries.

Fetal aspects-

- Baby born at term from severely anemic mother will not be anemic at birth. But there is little or no reserved iron. So anemia develops at neonatal period,

- Preterm labour,

- LBW (Low Birth Weight),

- IUD (Intra-Uterine Death).

\section{Discussion}

The most common cause of anemia in pregnancy is iron deficiency. It usually occurs due to low iron stores prior to pregnancy. The growing fetus depletes what stores there are and takes priority for any iron available. It is important to remember that increased iron requirements continue after the birth of the baby due to blood loss and breastfeeding. Less often, anemia in pregnancy is caused by folic acid deficiency. In certain populations, pregnancy can be complicated by sickle cell trait and anemia, as well as thalassaemias ${ }^{11}$. These diseases, in which the red blood cells are abnormal, present special problems in pregnancy. In some populations, as many as $80 \%$ of pregnant women are anemic. They are generally women from lower socio-economic groups in developing countries as well as pregnant teenagers. Women who experienced heavy periods and those who became pregnant soon after the birth of a child are at particular risk of becoming anemic in pregnancy. The symptoms such as tiredness and general weakness will be similar to those of any other type of anemia. In severe cases, the woman will be short of breath even at rest. If the anemia is prolonged, other signs of iron-deficiency anemia may develop such as a smooth shiny tongue and tenderness of the skin at the corners of the mouth. However, these advanced signs are rare. The diagnosis is made by examining a full blood count and noting the low hemoglobin concentration as well as the characteristic small, pale red blood cells under the microscope (in the case of iron deficiency anemia). The diagnosis of iron deficiency anemia can be confirmed by measuring the 
amount of storage iron as well as the levels of iron binding proteins in the blood. The diagnosis of folate deficiency is confirmed by estimating the red blood cell folate levels. If the anemia does not respond to iron treatment, additional folic acid deficiency should be suspected. A well balanced diet is always recommended but iron and folate supplementation is indicated in pregnancy. When the anemia is caused by lack of iron, it is treated with iron supplements, preferably ferrous sulphate tablets $(300 \mathrm{mg})$. These supplements should not be taken more than twice daily, since the side effects of iron are increased in doses of more than two daily. The side effects are stomach upsets and constipation which are problematic in pregnancy ${ }^{12}$. If the anemia is due to folic acid deficiency, it is treated with folic acid supplements ( 1 to $5 \mathrm{mg}$ once a day). As long as the anemia is treated and corrected, there should be no problem.

In majority cases, the management of anemia in pregnancy is easy \& worthwhile if the pregnant women $\&$ their guardians remain alert to protect the health of mother $\&$ baby. They need to ensure proper antenatal check up, perinatal \& postnatal cares \& follow relevent medical advice.

All pregnant women should be fully assessed at the start of their pregnancy so that any problems such as anemia will be picked up and treated.

\section{References}

1. Centres for Disease Control and Prevention. Recommendations to Prevent and Control Iron Deficiency in the United States. Morbidity and Mortality Weekly Report. 1998;47(No. RR-3)

2. Breymann C, Bian X, Blanco-Capito LR, et al. Expert recommendations for the diagnosis and treatment of iron-deficiency anemia during pregnancy and the postpartum period in the Asia-Pacific region. Journal of Perinatal Medicine. 2010;38:1-8.

3. Milman N. Prepartum anaemia: prevention and treatment. Annals of Haematology. 2008;87:949-59.
4. Reveiz L, Gyte GMI, Cuervo LG. Treatments for iron-deficiency anaemia in pregnancy. The Cochrane Database of Systematic reviews. 2007(2).

5. Johnson TA. Anaemia. In: Luesley DM, Baker PN, editors. Obstetrics and Gynaecology An evidence-based text for MRCOG 2nd ed. London: Hodder Arnold; 2010;139-43.

6. Pavord S,Myers B, Robinson B, Allard S, Strong J, Oppenheimer $\mathrm{C}$. UK guidelines on the management of iron deficiency in pregnancy. $\mathrm{Br} \mathrm{J}$ Haematol. 2012; 156:588-600.

7. South Australian Perinatal Practice Guidelines Chapter 60 Anaemia in pregnancy. South Australia [Updated 2012 May 22; cited 2013 December 17]. Available from:http://www.health.sa.gov.au/ppg/Default. aspx?tabid $=95$

8. Milman N. Iron and pregnancy -a delicate balance. Annals of Hematology. 2006;85:559-65.

9. Pasricha SRS, Flecknoe-Brown SC, Allen KJ, et al. Diagnosis and management of iron deficiency anaemia: a clinical update. MJA. 2010;193:525-32.

10. The American College of Obstetricians and Gynecologists. ACOG Practice Bulletin No. 95 Anemia in Pregnancy. Obstetrics \& Gynecology. 2008;112:2017.

11. Bryant C, Larsen S. Anaemia in pregnancy. The Australian and New Zealand Journal of Obstetrics and Gynaecology. 2009;11:17-8.

12. Simpson JL, Bailey LB, Pietrzik K, et al. Micronutrients and women of reproductive potential: required dietary intake and consequences of dietary deficiency or excess. Part 1 -Folate, Vitamin B12, Vitamin B6. J Matern Fetal Neonatal Med. 2010;23:1323-43. 